\title{
REGULARIZAÇÃO DE VAZÃO E DISSIPAÇÃO DE ENERGIA EM BACIAS HIDROGRÁFICAS NA MESORREGIÃO CENTRO OCIDENTAL RIO- GRANDENSE
} \author{
Vitória KROLOW ${ }^{1}$ \\ capoane@gmail.com; daniela21.vk@gmail.com \\ jwrasche@yahoo.com.ar

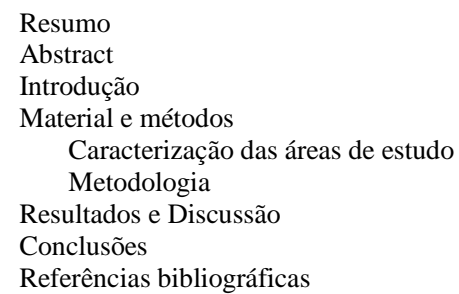

FLOW REGULARIZATION AND POWER DISSIPATION IN WATERSHED IN THE CENTER WEST REGION OF RIO GRANDE DO SUL STATE, BRAZIL

Danilo Rheinheimer dos SANTOS ${ }^{1}$, André PELLEGRINI ${ }^{2}$, Jimmy Walter Rasche ALVAREZ ${ }^{3}$, Ivan Renato Cardoso KROLOW ${ }^{1}$, Viviane CAPOANE ${ }^{1}$, Daniela da Rocha

(1) Universidade Federal de Santa Maria, Departamento de Solos. E-mails: danilonesaf@gmail.com; ivanrk.rk@ibest.com.br;

(2) Universidade Tecnológica Federal do Paraná, Campus Dois Vizinhos. E-mail: andre.pellegrini@ yahoo.com.br

(3) Universidad Nacional de Asunción, Facultad de Ciencias Agrarias, Departamento de Suelo y Ordenamiento Territorial. E-mail:

RESUMO - As ações antrópicas determinam as condições de superfície e alteram as proporções de uso e manejo do solo em bacias hidrográficas. $\mathrm{O}$ uso de coeficientes que consideram variáveis hidrossedimentológicas para avaliar a interação homem-meio pode ser a forma eficaz de quantificar os impactos da ação do Homem no meio. O objetivo deste trabalho foi avaliar as condições de superfície proporcionadas pela interação homem-meio através da regularização de vazão e da dissipação de energia em duas pequenas bacias hidrográficas em áreas de assentamentos de reforma agrária (diferentes formas de proteção das áreas ripárias) no Rio Grande do Sul. Na pequena bacia com maior cobertura por mata ciliar, os coeficientes apontaram para maior regularização da vazão, porém menor dissipação de energia erosiva. A degradação dos estratos inferiores da mata (acesso livre aos bovinos) compromete a capacidade de retenção de sedimentos comparativamente a outra pequena bacia, cujas regiões próximas aos cursos d'água predominam áreas úmidas. $\mathrm{O}$ escoamento superficial gerado nas áreas de lavoura foi atenuado pela área de mata na bacia maior $\mathrm{e}$ potencializado pelas áreas úmidas na bacia menor.

Palavras-chave: produção de sedimento, erosão, zona ripária.

ABSTRACT - Anthropogenic actions determine surface conditions and alter the proportions of land use and management in river basins. The use of coefficients that consider hydrosedimentological variables to evaluate man-environment interaction can be the effective way of quantifying the impacts of human action in the environment. The objective of this work was to evaluate the surface conditions provided by man-environment interaction through the regularization of flow and energy dissipation in two small river basins in areas of agrarian reform settlements (different forms of protection of riparian areas) in Rio Grande do Sul. In the small basin with greater coverage by riparian forest, the coefficients pointed to a greater regularization of the flow, but less dissipation of erosive energy. The degradation of the lower strata of the forest (free access to the cattle) compromises the capacity of retention of sediment compared to another small basin, whose regions near the watercourses predominate wetlands. The surface runoff generated in the cropping areas was attenuated by the forest area in the larger basin and enhanced by the humid areas in the lower basin. Keywords: sediment production, erosion, riparian zone.

\section{INTRODUÇÃO}

A visão centrista do Homem sobre a natureza e a explosão no crescimento populacional aceleraram o domínio e a destruição dos biomas naturais. Após a revolução verde e, mais recentemente, a biotecnológica simplificaram-se os sistemas produtivos e maximizaram-se os distúrbios ambientais, mesmo nos ecossistemas já cultivados. Conhecimentos até então consolidados entre os técnicos, como a aptidão de uso do solo, estão sendo relativizados e até negligenciados.
O uso do solo sem considerar sua aptidão agrícola e com manejo inadequado está gerando graves problemas ambientais, principalmente, àqueles relacionados ao escoamento superficial de águas pluviais. Segundo Oliveira et al. (2013), a utilização das terras e o crescimento econômico têm sido constantemente prejudicados pela falta de planejamento, que tenha como base o conhecimento dos recursos naturais disponíveis. Na região do planalto do Rio Grande do Sul, mais de $90 \%$ dos biomas naturais já foram convertidos em agroecos- 
sistemas, principalmente pelas commodities soja e milho (Gubiani et al., 2015). Segundo Ziech et al. (2015), no ano agrícola 2012/2013, $31 \%$ e $69 \%$ do solo das áreas cultivadas com milho e soja apresentava menos do que $40 \%$ da superfície coberta por resíduos da espécie antecessora, aquém do minimamente aceitável para o bom funcionamento do sistema plantio direto. Embora sejam inquestionáveis as vantagens do sistema plantio direto na minimização dos danos ambientais, são percebidos vários equívocos: a baixa adoção da rotação de culturas, a manutenção de pouca palha em superfície, a compactação do solo, a retirada de terraços, a semeadura morro abaixo, a aplicação de fertilizantes como fósforo e potássio em superfície, entre outros de menor influência (Denardin et al., 2005).

A mensuração dos impactos das atividades agropecuárias sobre a transferência de solo e de água para os mananciais aquáticos pode ser feita por diferentes modelos matemáticos. Indubitavelmente, a Equação Universal de Perda de Solos (USLE) é a mais conhecida e ainda amplamente utilizada. Exemplo disso é que, em 2015, Miqueloni, Gianello e Bueno, a usaram para estimar as perdas de solo e a identificação dos fatores que mais contribuem na degradação ambiental. Porém, Odongo et al. (2013) relatam que a USLE é mais adequada para avaliar perdas de solo em áreas pequenas. Seu emprego em grandes áreas aumenta a margem de erros, principalmente por não considerar diretamente processos físicos envolvidos na erosão como o escoamento superficial. Para áreas em escala de bacias, há vários modelos matemáticos de reprodução dos fluxos hidrológicos: SWAT, LISEM, WATEM/SEDEM, ARS, SWRRB, CREAMs, GLEAMs, dentre outros, que possibilitam quantificar as perdas de solo e de poluentes e prever cenários futuros através de simulações (Bressiani et al., 2015; Garbossa et al., 2011; Perazzoli et al., 2013; Santos, et al., 2010). Os modelos matemáticos simplificados como os coeficientes de regularização de vazão e de dissipação de energia erosiva aportam informações relevantes sobre os locais prioritários para a tomada de medidas preventivas e corretivas em escala de bacias hidrográficas (D'Agostini, 1999; D’Agostini \& Martini, 2000). Esses são utilizados de forma comparativa, no intuito de avaliar a relação homem-meio, em estudos de bacias hidrográficas.

Em consonâncias com outras atividades que vem sendo realizadas de forma participativa com os técnicos e assentados de reforma agrária, esse trabalho visa utilizar os coeficientes de regularização de vazão e de dissipação de energia erosiva para evidenciar a importância da preservação da mata ciliar e das áreas úmidas entorno das pequenas bacias hidrográficas.

\section{MATERIAIS E MÉTODOS}

\section{Caracterização das áreas de estudo}

As duas pequenas bacias hidrográficas (PBH's) monitoradas estão localizadas no Assentamento de Reforma Agrária Alvorada, no município de Júlio de Castilhos, Região Geomorfológica do Planalto das Missões do estado do Rio Grande do Sul, Brasil.

A área total do assentamento é de 1569 ha. As áreas das duas PBH's têm 144,5 ha e 79,6 ha e foram denominadas como PBH 140 e PBH 80, respectivamente. As PBH's fazem parte da bacia hidrográfica do rio Jacuí e são consideradas de terceira ordem.

O clima da região é subtropical úmido com verões quentes e invernos com geadas frequentes.

A precipitação média anual histórica entre os anos de 1972 a 2002 foi de $1.639 \mathrm{~mm}$, com chuvas bem distribuídas ao longo do ano (Agritempo, 2013).

O solo predominante é o Argissolo, com textura média para o horizonte A apresentando $(67,2 \%$ de areia, $17,4 \%$ de silte e $15,4 \%$ de argila).

\section{Metodologia}

$\mathrm{Na}$ Figura 1 estão plotados os mapas de distribuição de usos da terra no período monitorado e explicitado nesse artigo.

O mapa de uso foi gerado utilizando uma imagem pancromática do satélite QuickBird com $60 \mathrm{~cm}$ de resolução de pixel.

Nas PBH's predominam a integração lavoura-pecuária, com média de 75,5\% da área com cultivo da soja e do milho no verão e pastagens no inverno para bovinocultura de leite. 

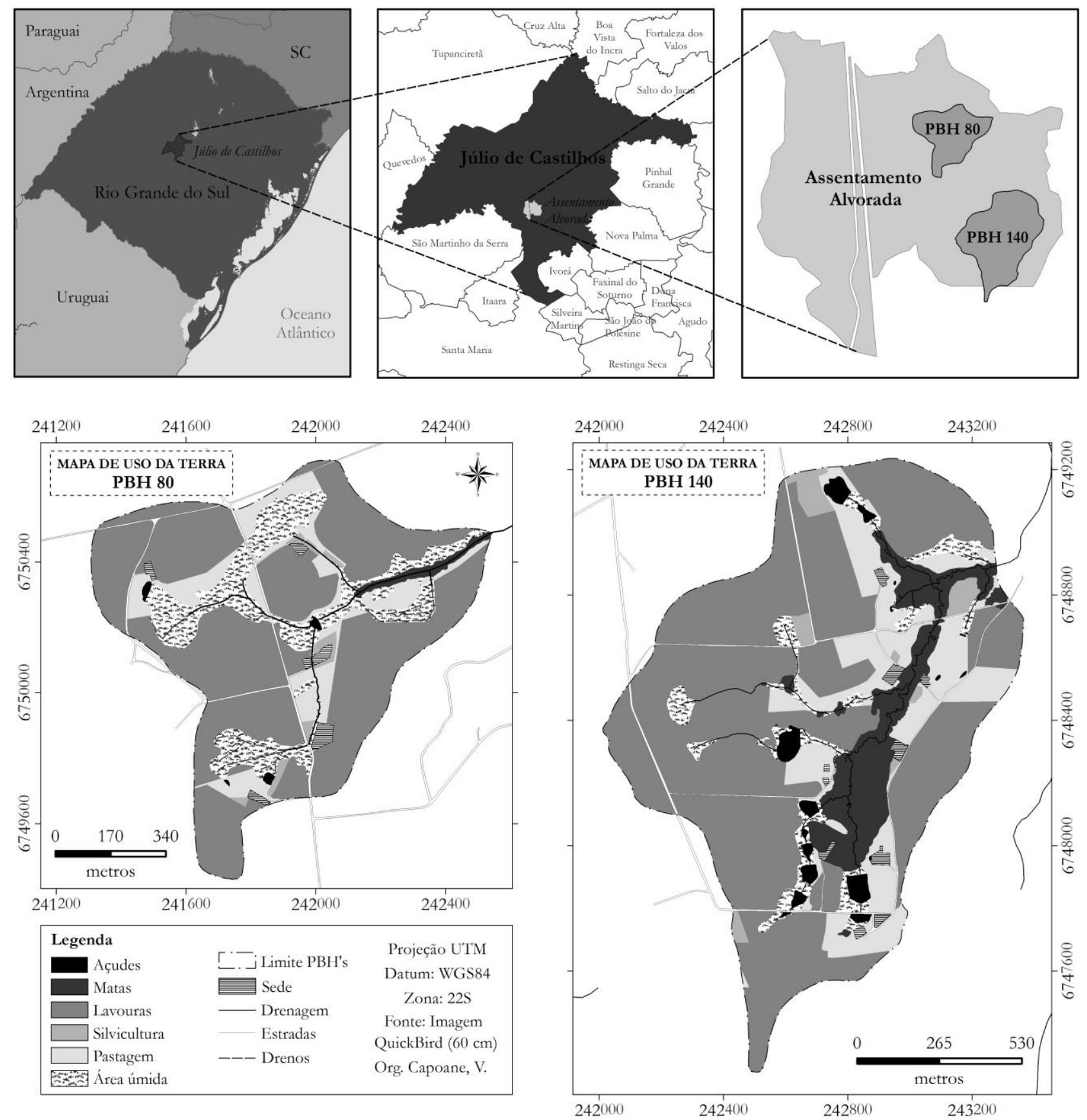

Figura 1. Localização das áreas estudadas e distribuição de usos do solo em 2010 e 2012 nas duas bacias hidrográficas (PBH 140 e PBH 80). Assentamento de reforma agrária Alvorada, Júlio de Castilhos, Rio Grande do Sul, Brasil.

As principais diferenças entre as duas PBH's, dada ênfase no presente estudo, são a área com mata ciliar $(12,2 \%$ na $\mathrm{PBH} 140 \mathrm{e}$ $2,4 \%$ na $\mathrm{PBH} 80)$ ou por áreas úmidas $(15,3 \%$ na $\mathrm{PBH} 80$ e 5,3\% na PBH 140) e a declividade. As classes de declividade e a área relativa das $\mathrm{PBH} 80$ e $\mathrm{PBH} 140$ foram respectivamente: $0-3 \%=10,1$ e 10,3 ha; $3-6 \%$ $=20,6$ e 27,3 ha; $6-9 \%=17,7$ e 32,2 ha; $9-12 \%$ $=14,3$ e 31,2 ha; $12-15 \%=16,7$ e 43,5 ha. $\mathrm{O}$ perímetro e as diferenças de cotas das duas PBH's foram 4.171,3 m e 431 - 485 para a PBH
80 e 5.308,4 m e 440 - 506 m para a PBH 140. O comprimento da rede de drenagem (Lw), o comprimento médio da rampa (L) e o seno da inclinação média ponderada $(\operatorname{sen} \theta)$ foram 3.876,00 m, 51,5 m e 0,077 para PBH 80 e $5.118,90 \mathrm{~m}, 71,0 \mathrm{~m}$ e 0,089 para a $\mathrm{PBH} 140$, respectivamente. As informações sobre o relevo foram extraídas do modelo digital de elevação de $5 \mathrm{~m}$ de resolução de pixel, gerado com dados de levantamento planialtimétrico com aparelho de posicionamento cinemático em tempo real (RTK). 
$\mathrm{O}$ fator de forma (Kf) foi calculado pela divisão entre o comprimento médio da subbacia e o comprimento axial; enquanto que o coeficiente de compacidade $(\mathrm{Kc})$ foi obtido pela divisão entre o perímetro real da sub-bacia e o perímetro de um círculo imaginário com área igual a da sub-bacia. Os dados de Tempo de Concentração médio ( $\mathrm{Tc}_{\text {Médio }}$ ) e Tempo de pico médio (Tppédio) foram calculados nas duas PBH's ao serem analisados 196 eventos pluviométricos.

O monitoramento dos atributos hidrossedimentológicos foi realizado durante 36 meses (janeiro de 2010 a dezembro de 2012), avaliando-se 205 eventos pluviais. Os dados pluviométricos foram obtidos de dois pluviógrafos (um em cada bacia) e três pluviômetros instalados nas duas PBH's.

$\mathrm{Na} \mathrm{PBH} 80$ o pluviógrafo foi conectado a um datalogger (SL PNV 2000), que registrava os dados em intervalos de 5 minutos, enquanto que na $\mathrm{PBH} 140$ próximo ao exutório, foi instalada uma estação meteorológica automática (Danvis, Vantage Pro 2), que captava leituras em intervalos de 10 minutos.

$\mathrm{O}$ trabalho erosivo $\left(\mathrm{W}_{\mathrm{E}}\right)$ foi obtido a partir do cálculo da produção de sedimento (PS) de cada $\mathrm{PBH}$, em cada evento erosivo. Procedeuse no somatório da produção de sedimentos em suspensão (PSS) e da produção de sedimentos transportados (PST) em arraste de fundo.

A concentração de sedimento em suspensão (CSS) foi determinada pelo método da evaporação em estufa a $105^{\circ} \mathrm{C}$. Com os resultados de CSS foram geradas equações através de relações com os valores de turbidez (NTU - Nephelometric Turbidity Unit) e também com os valores de vazão.

O monitoramento da vazão se deu através de duas calhas Parshalls com larguras críticas de 1,22 e 1,52 m, para a PBH 80 e PBH 140, respectivamente. Ambas as variáveis foram registradas constantemente por sensores de turbidez e de nível acoplados a um datalogger que registrou os valores em intervalos de 5 minutos.

A PSS, nos diferentes períodos climáticos, foi obtida pela média dos resultados da aplicação de equações nos valores contínuos de vazão e turbidez. Por meio destes pontos foi construída, para cada $\mathrm{PBH}$, uma curva-chave a qual relaciona a massa de sedimento transportada no tempo e em diferentes vazões. Estas equações foram aplicadas nos valores contínuos de vazão resultando na descarga de sedimento, que posteriormente foi multiplicado pelo tempo, obtendo-se assim a PST por arraste de fundo.

A separação do escoamento foi feita usandose o método descrito por Tucci (2002). Para obter os valores de energia cinética de cada evento considerou-se a intensidade da precipitação (Wischmeier \& Smith, 1978).

As condições de manejo e cobertura vegetal do solo foram avaliadas utilizando o Coeficiente de Regularização de Vazão (CRV) descrito por D'Agostini \& Martini (2000). O CRV arquiteta um coeficiente capaz de quantificar a eficiência de fatores que determinam as flutuações de vazão (Equação 1). Seus termos podem ser descritos, através do resultado matematizado do coeficiente da eficiência de fatores mutáveis pela ação humana $\left(\beta_{h s}\right)$ capazes de produzir flutuações de vazão $\left(Q\left(\mathrm{~m}^{3} \mathrm{~s}^{-1}\right) ; D\right.$ e $\left.t(\mathrm{~s})\right)$, através da interação do volume precipitação em determinado tempo $\left(i\left(\mathrm{~m} \mathrm{~s}^{-1}\right)\right)$, sobre uma bacia hidrográfica com características imutáveis de área $\left(\mathrm{A}\left(\mathrm{m}^{2}\right)\right)$ e padrão geométrico $\left(\theta_{\lambda}(\mathrm{m})\right)$.

$$
\beta_{h s}=\frac{\sqrt{\frac{\sum_{j=1}^{n}\left(Q_{j}-Q\right)^{2} D_{\Delta Q j}}{n}}}{\sqrt{\frac{\sum_{k=1}^{n}\left[\left(i_{k}-i_{m}\right) A\right]^{2} \Delta t_{k}}{n} \operatorname{Sen}^{-1} \theta_{\lambda}}}
$$

$$
\text { O CRV é obtido pela fórmula: }
$$

$$
C R V=1-\beta_{h s}
$$

Então, valores próximos de um $(\mathrm{CRV}=1)$ são considerados favoráveis de regularização de vazão.

O coeficiente beta $(\beta)$ pode ser descrito em seus termos, por meio da razão entre o $\left(\mathrm{W}_{\mathrm{E}}(\mathrm{J})\right)$ e o somatório correspondente à energia cinética da massa da chuva incidente (Ecp (MJ)), com o produto entre $(\mathrm{M}=$ lâmina de chuva $(\mathrm{m}) *$ massa específica $(\mathrm{kg}) *$ área da bacia $\left(\mathrm{m}^{2}\right)$ ) com a aceleração da gravidade $\left(\mathrm{g}\left(\mathrm{m} \mathrm{s}^{-1}\right)\right)$, o comprimento médio da rampa $(\mathrm{L}(\mathrm{m}))$ e o seno ao quadrado da inclinação média ponderada $(\theta$ (grau)).

$$
\beta=\frac{W_{E}}{\left(E c p+M g L \operatorname{Sen}^{2} \theta\right)}
$$


O Coeficiente de Dissipação de Energia Erosiva (CDEE - D’Agostini, 1999) é, finalmente, obtido pela equação:

$$
\mathrm{CDEE}=1-\beta
$$

Então, valores próximos de um $(\mathrm{CDEE}=1)$ são considerados favoráveis à preservação do solo.

A correlação entre a precipitação total (PT) e o escoamento superficial (ES) foi obtida pelos cálculos de 205 eventos pluviométricos e os o valores de CRV, W(kj), PS ( $\left.\mathrm{kg} \mathrm{ha}^{-1}\right), \mathrm{EC}(\mathrm{MJ})$, MgLSen $01\left(\mathrm{MJ} \mathrm{ha}^{-1}\right)$ e CDEE foram obtidos de 119 eventos ocorridos em ambas PBH's. Os valores de ES, de CRV e de potencial energético foram correlacionados com os valores de precipitação. Os valores de $\mathrm{W}_{\mathrm{E}}$ foram correlacionados com os valores de potencial energético.

Os valores e CRV e CDEE das duas bacias foram correlacionados entre si. Os dados utilizados na obtenção de CDEE foram previamente transformados em $\log _{10}$ e com as demais variáveis foram correlacionados por equação polinomial de primeiro grau, com $95 \%$ de intervalo de confiança (Sistema de Análises Estatística - Winstat - Machado, 2001).

\section{RESULTADOS E DISCUSSÃO}

As correlações entre a PT e o ES não foram significativas para as duas PBH's (Figura 2).

Em janeiro de 2010 a precipitação pluvial foi de $676 \mathrm{~mm}$, cuja média mensal dos últimos 30 anos é de apenas $137 \mathrm{~mm}$. Em dois eventos pluviométricos (322, 9 e 157,9 $\mathrm{mm}$ ) os valores do ES foram de 88,5 e 39,1 mm. Esses valores

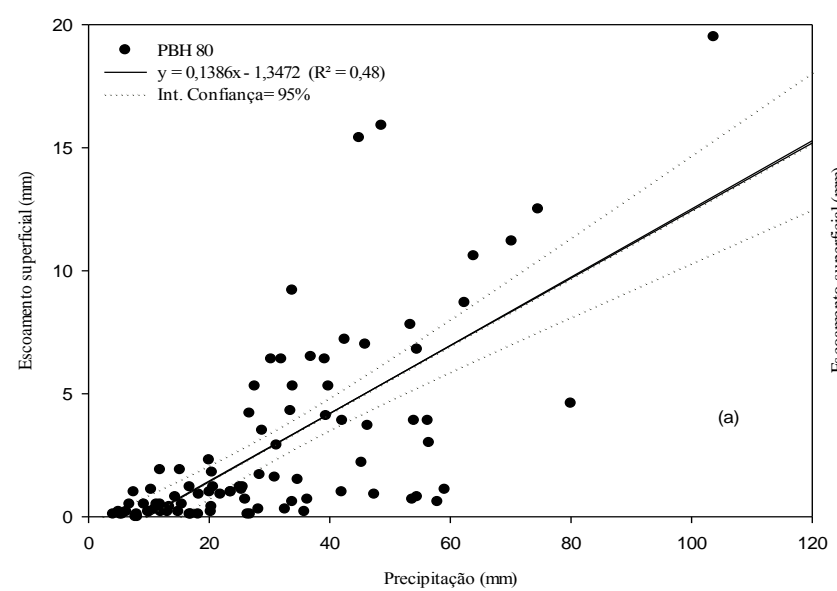

Figura 2. Escoamento superficial em função da precipitação total nas pequenas bacias hidrográficas PBH 80 (a) e PBH 140 (b) no período de agosto de 2010 a dezembro de 2012.

Os valores das equações plotadas (Figura 2, a e b) foram positivos com coeficientes de determinação baixos (48\% e 39\%). Embora as PBH's sejam consideradas pareadas, a dispersão dos pontos na PBH 140 mostra se superior, o que demonstra a acurácia ligeiramente superior da PBH 80. Há tendência positiva do ES com a menor dispersão de pontos, variando entre o mínimo de 4,1 mm e o máximo de $103,7 \mathrm{~mm}$ (Média $=30,4 \mathrm{~mm} ; \sigma=19,58)$ na $\mathrm{PBH} 80$, assim como na PBH 140 que apresentou valor mínimo de 4,8 mm e o máximo de 105,9 mm (Média $=32,4 ; \quad \sigma=19,90$ ). $\mathrm{A}$ menor significância dos valores encontrados na $\mathrm{PBH}$ são extremamente elevados e anormais para essas duas PBH's e foram excluídos da análise de correlação. Além disso, durante esses eventos somente foram monitorados os parâmetros hidrossedimentológicos da $\mathrm{PBH}$ 140, impedindo o comparativo entre as duas bacias, objeto principal desse trabalho.

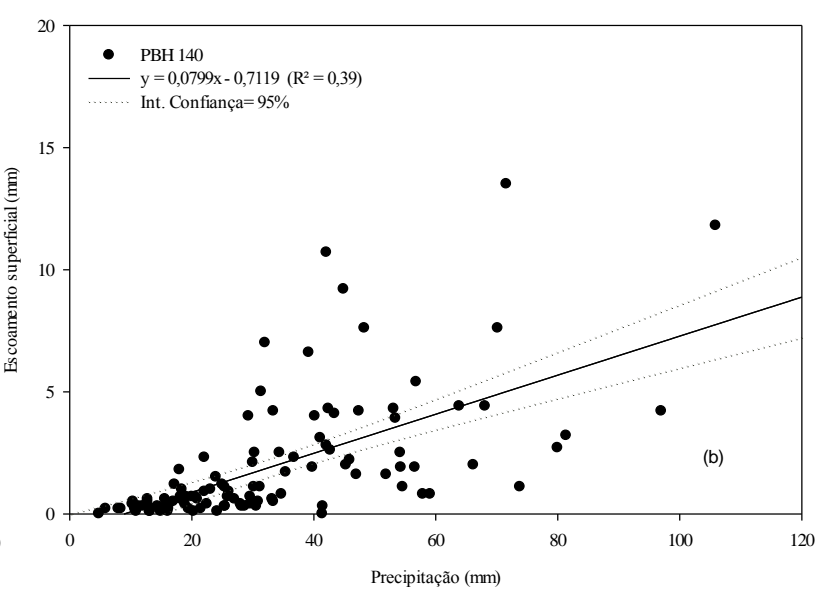

140 esta relacionado aos fatores como da maior irregularidade superficial do terreno, maior cobertura de solo, representada pelas matas ciliares, o que favorece a rugosidade da área avaliada em prol da redução do ES.

Resultados que corroboram com Souza et al. (2015) que encontraram relação positiva do escoamento superficial com a precipitação, assim como relação direta com a vegetação em precipitações de mesma intensidade.

A relação positiva da PT com o ES se deve a condição de forma e relevo da PBH's que são de tendência mediana a enchentes conforme foi constatado através dos valores de $\mathrm{Kc}$ que 
corresponderam a 1,34 e 1,31 para as $\mathrm{PBH} 80 \mathrm{e}$ $\mathrm{PBH}$ 140, respectivamente; assim como o $\mathrm{Kf}$ com 0,47 (PBH 80) e 0,45 (PBH 140). A forma alongada semelhante de ambas PBH's as tornam mais complexas na sua avaliação a fim de identificar variáveis de maior e de menor impacto nos processos erosivos.

No entanto, os dois coeficientes corroboram de que as duas bacias não são sujeitas ou são de tendência mediana a grandes enchentes (Lima et al., 2013; Rocha et al., 2014; Tonello et al., 2006). Durante os 36 meses de monitoramento foi constatada apenas uma enchente de grandes proporções $(322,9 \mathrm{~mm}$ ocorrido no início de janeiro de 2010).

Andrade et al. (2008) ao investigarem uma bacia do Centro Sul Mato-grossense confirmaram a baixa suscetibilidade a enchentes em condições normais de precipitação, excluindo-se eventos de intensidades anormais, pelo fato de o Kc apresentar o valor afastado da unidade $(1,43)$ e, quanto ao seu fator de forma, exibir um valor baixo $(0,21)$.
As duas PBH's monitoradas são de terceira ordem e as variáveis hidrossedimentológicas estão muito sensíveis aos efeitos diretos do uso da terra (Mioto et al., 2014).

Nesses casos, como relatado por Bias et al. (2012), há relação entre escoamento superficial e potencial erosivo definido com a interceptação vegetal.

Quanto maior for a interceptação vegetal, menor será a quantidade de água que atinge o solo e, portanto, menor será o ES e a possibilidade de erosão do solo.

O valor médio do tempo de concentração (Tc) e do Tempo de pico (Tp) nas duas bacias corresponderam a $84 \mathrm{~min}(\sigma=36,20)$ e $210 \mathrm{~min}$ $(\sigma=76,79)$ para $\mathrm{PBH} 80$ e de $58 \mathrm{~min}(\sigma=$ 21,33) e $141 \min (\sigma=44,73)$ PBH140, respectivamente. $\mathrm{O}$ menor $\mathrm{Tc}$ na $\mathrm{PBH} 80$ esta diretamente ligado ao maior $\mathrm{Kf}$, menor diferença de cotas $(54 \mathrm{~m})$, e distribuição e uso do solo em relação a PBH 140.

Houve grande dispersão dos dados de CRV (Figura 3a e 3b).
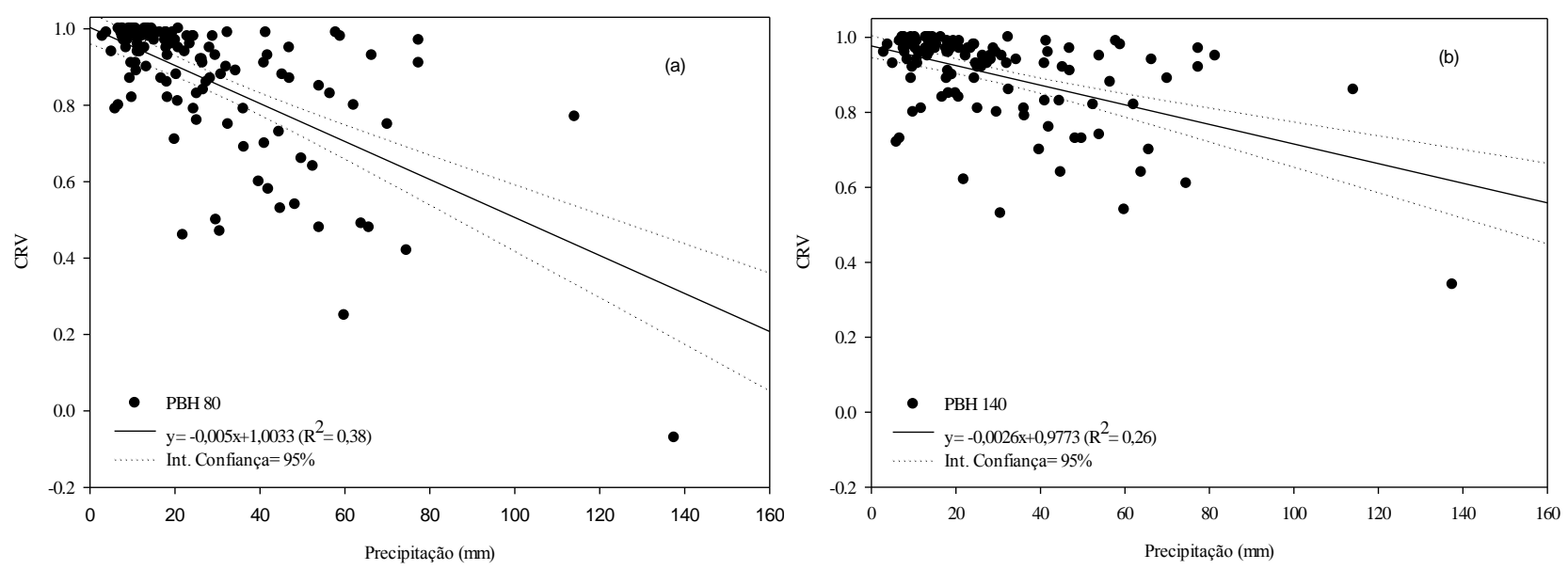

CRV (PBH 80)

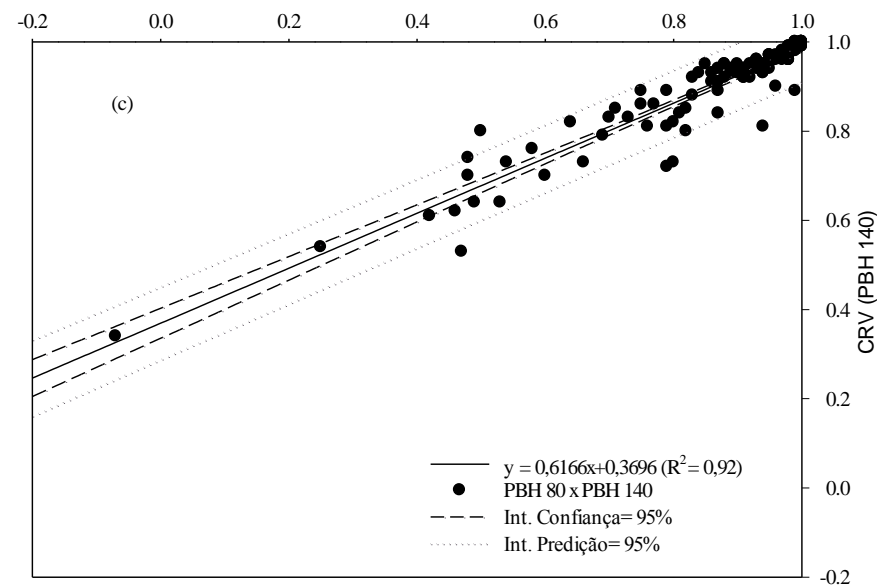

Figura 3. Coeficientes de regularização de vazão (CRV) em função da precipitação no período de agosto de 2010 a dezembro de 2012 (a e b); e relação do CRV entre as PBH's 80 e 140 (c). 
Contudo, a relação foi significativa (p>0,001). Na PBH 80 a tendência de maior redução do CRV com incremento dos volumes precipitados (Figura 3a). Também se obteve menor diferença nos valores de CRV, principalmente em precipitações menores de 30 $\mathrm{mm}$ e com baixa intensidade. Os valores de CRV variaram de $-0,07$ a 1 (Média $=0,86 ; \sigma=$ 0,18 ) na PBH 80 e de 0,34 a 1 (Média $=0,90 ; \sigma$ $=0,12)$ na $\mathrm{PBH} 140$. O maior valor encontrado na PBH 140 confere melhor regularização de vazão, uma vez que se apresenta mais próximo da unidade (Figura 3b).

Um dos fatores que possivelmente contribuiu para o menor valor de CRV na PBH 80 se deve ao maior volume precipitado no mês de agosto que atingiu 193,7 mm (distribuídos em 12 eventos pluviométricos), três a mais do que na $\mathrm{PBH} 140$ que acumulou $181,5 \mathrm{~mm}$ (distribuídos em nove eventos pluviométricos). Comparando-se os resultados, percebe-se que a melhor distribuição das chuvas na PBH 80 não foi suficiente para um CRV mais próximo de um, assim como a proximidade das duas PBH's não exclui a probabilidade de ocorrência diferenciada de eventos pluviométricos capazes de desencadearem os processos erosivos.

A melhor distribuição das águas pluviais não proporcionou resultados mais favoráveis para a PBH 80 no que tange ao ES (Média = 2,86; $\sigma=$ 3,90 ) durante o período (Figura 2), uma vez que atingiu valor superior em 34,3\% ao da $\mathrm{PBH} 140$ (Média $=1,88 ; \sigma=2,52$ ) (Figura 3b). Essa informação é pertinente na justificativa do menor CRV, porém refuta o comportamento pluvial semelhante, o que enfatiza a complexidade das PBH's monitoradas. Observou-se alta correlação entre os valores de CRV das duas PBH's (Figura 3c), com resultados mais favoráveis para a regularização de vazão na PBH 140 (mais próximos de um), do que na PBH 80.

Na PBH 140 é constatado maior área com declividade entre 9 e $15 \%$ (74,7 ha) do que na PBH 80 (31 ha) da mesma forma que apresenta diferenças entre cotas igual a $66 \mathrm{~m}$. Essa faixa de declividade considera os locais avaliados como de relevo ondulado, classificação que poderia induzir a afirmação de que os CRVs seriam semelhantes em ambas PBH's, o que é refutado diante da análise. Nesse sentido, outros fatores como a distância percorrida pelas águas pluviais até o leito único da PBH 140, ocupação das áreas por matas e cultivo de milho e soja e ocupação das áreas por banhados podem estar contribuindo de maneira mais efetiva com o CRV. Na PBH 140 são distribuídas entre áreas de mata, cultivo de milho e soja 89,9 ha, enquanto na PBH 80, 60,8 ha. Por outro lado, se há maior área de exploração na PBH 140, há menor área úmida (banhado) do que na $\mathrm{PBH}$ 80, essa, inferior em 4,51 ha. Valores distintos também são obtidos nas áreas de matas e de silvicultura que totalizam 15,7 ha a mais na PBH 140 do que na PBH 80.

As diferenças encontradas no uso do solo associadas ao manejo das áreas esta contribuindo decisivamente com o menor valor de CRV da PBH 80, principalmente naquelas áreas cultivadas com o milho e a soja, que embora se assemelhe em ambas as PBH's por serem utilizados na entressafra para o pastoreio dos animais, tem sua ocupação superior a PBH 140. O impacto físico do pisoteio na camada superficial do solo se agrava em áreas com menor capacidade de infiltração e consequentemente maior ES, o que se mostrou mais perceptível na $\mathrm{PBH}$ 80, principalmente em função da ocupação por áreas úmidas.

A taxa de infiltração pluviométrica é reduzida em função do pastoreio intenso e agrava-se com a carga excessiva, aumentando assim o ES. Lanzanova et al. (2007) ao avaliarem a importância da cultura antecessora às pastagens de inverno, na taxa de infiltração de água no solo, constataram maiores evidências nas áreas cultivadas com milho, em comparação à soja. Os autores consideraram que os resíduos de milho foram suficientes para prevenir prejuízos à infiltração de água induzidos pela frequência de pastejo e o inverso na cultura da soja, mesmo ao reduzir a frequência de pastejo. Contudo, as condições de superfície que mais influenciaram no CRV devem-se principalmente a maior porcentagem de área úmida existente na PBH 80 e a maior porcentagem de área de mata na PBH 140. Ambas as áreas se encontram posicionadas na paisagem abaixo das áreas de lavoura e próximas aos cursos d'água (Figura 1). Assim, o ES gerado nas áreas de lavoura foi atenuado pela área de mata e potencializado pelas áreas úmidas. Nesse sentido, reportou-se às considerações de Pereira \& Filho (2009), que 
consideram relação direta das áreas saturadas à erosão hídrica, pois os fluxos de águas superficiais e subsuperficiais se acumulam nas proximidades da drenagem e, dependendo da cobertura vegetal dessas áreas, ocorre a intensificação do carreamento das partículas de solo.

A relação da precipitação pluvial e o potencial energético (PE) (Figura 4a) apresentaram alto ajuste dos pontos.
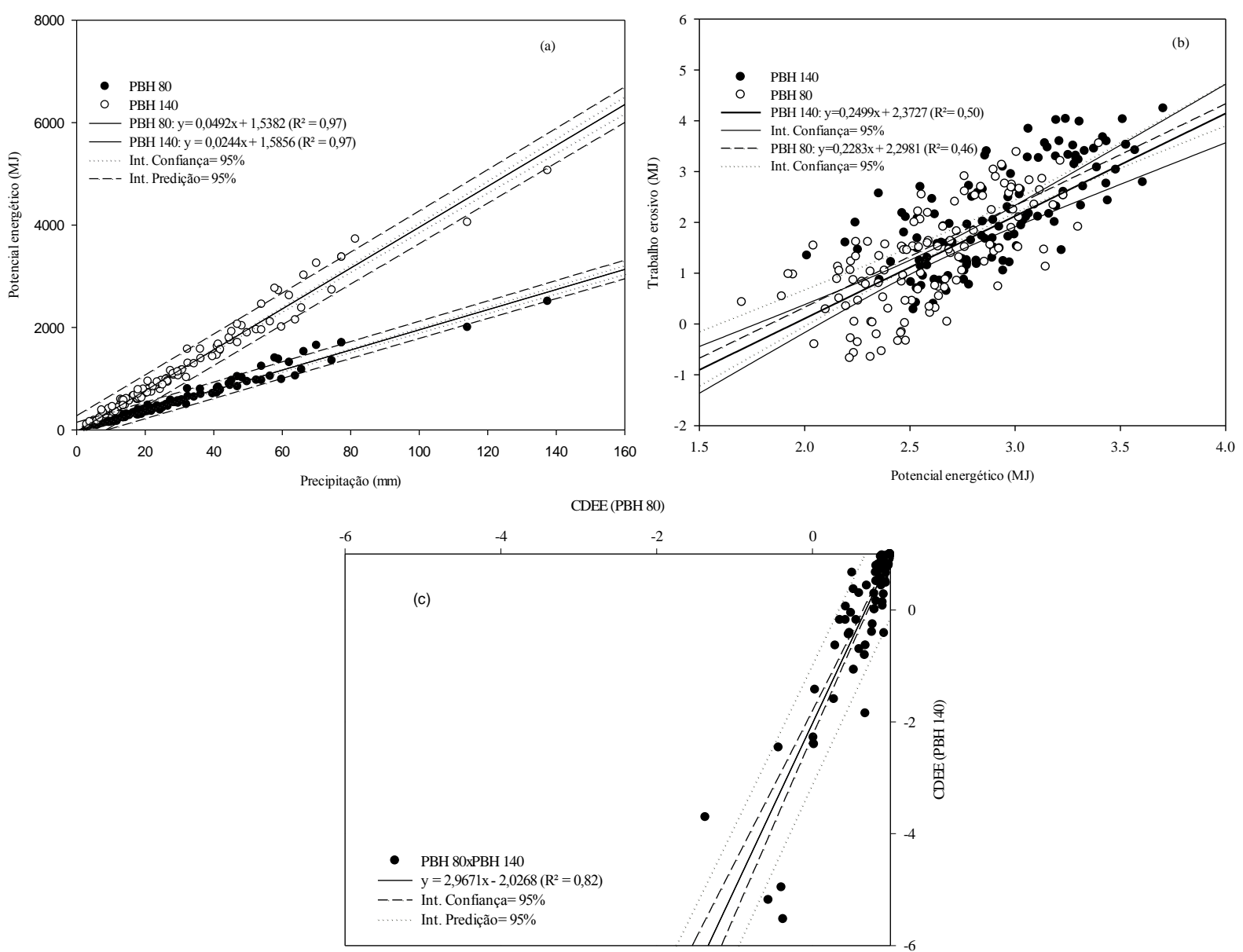

Figura 4. Relações da precipitação com potencial energético (a); do potencial energético e trabalho erosivo (b); e dos coeficientes de dissipação de energia erosiva da PBH 80 e da PBH140 (c).

$\mathrm{Na}$ PBH 140 houve maior incremento de energia potencialmente erosiva, com o aumento do volume precipitado. Na comparação das PBH's constatou-se que em precipitações a partir de $40 \mathrm{~mm}$ o PE aumentou consideravelmente, partindo de 1900 MJ até $5000 \mathrm{MJ}$, embora que o maior número de eventos tenha se concentrado abaixo desse ponto. Enquanto que na $\mathrm{PBH} 80$ o $\mathrm{PE}$ apresentou pico máximo em pouco mais que $2000 \mathrm{MJ}$, a diferença acentua-se principalmente a partir de $20 \mathrm{~mm}$ precipitados. A correlação entre os valores de PE com seus respectivos valores de trabalho $\left(\mathrm{W}_{\mathrm{E}}\right)$ foram ainda mais dispersos do que a quando da relação com potencial energético (Figura $4 b$ ).

$\mathrm{Na}$ Figura $4 \mathrm{c}$ observa-se que os valores CDEE das duas PBH's se correlacionam positivamente. Ao contrário do desempenho ocorrido com o CRV, o CDEE apresentou os piores indicadores para a PBH 140. Para cada KJ aplicado de energia erosiva resulta em 0,69 $\mathrm{J}$ de trabalho erosivo, o que confere CDEE de 0,31. Já na $\mathrm{PBH} 80$, para cada KJ de energia gera apenas $0,21 \mathrm{~J}$ de $\mathrm{W}_{\mathrm{E}}$, o que compreende a um CDEE de 0,79. Então, na PBH 140, o valor de CDEE foi 69,6\% maior do que na PBH 80. Esse incremento na taxa de dissipação de energia erosiva correspondeu a $0,48 \mathrm{~J} \mathrm{KJ}^{-1}$ e confere a $\mathrm{PBH} 140$ maior $\mathrm{W}_{\mathrm{E}}$.

O menor $\mathrm{W}_{\mathrm{E}}$ da $\mathrm{PBH} 80$ provavelmente está relacionado a maior presença de áreas úmidas (Figura 1), diferenciando-se na capacidade de retenção de parte dos sedimentos produzidos nas áreas de lavoura. Por outro lado, a menor eficiência da PBH 140 (menor CDEE) está 
relacionada a degradação da vegetação semiarbórea da mata ciliar devido ao livre acesso ao gado de leite. Isso atua de forma negativa na retenção dos sedimentos provenientes das lavouras.

Os coeficientes utilizados, com o objetivo de avaliar indiretamente as condições de superfície determinadas pela ocupação humana, apresentaram subsídios e identificaram fragilidades de uso e de manejo do solo, principalmente na PBH 80. A ação humana sobre estes coeficientes, apesar de subjetiva é de difícil quantificação, certamente determina a dinâmica hidrossedimentológica nas PBH's.

\section{CONCLUSÕES}

O uso dos coeficientes de regularização de vazão e de dissipação de energia erosiva é eficiente na avaliação dos processos erosivos das bacias hidrográficas.

Coeficiente de regularização de vazão é melhor na bacia hidrográfica com maior proporção de área de mata ciliar.

A manutenção de áreas úmidas na PBH 80, e o impedimento do acesso aos animais na mata ciliar na PBH 140, são medidas eficazes para minimizar os danos provocados pelo escoamento superficial.

O coeficiente de dissipação de energia erosiva indica maior trabalho erosivo na bacia hidrográfica com maior proporção de área de mata ciliar.

O coeficiente de dissipação de energia erosiva indica que na bacia hidrográfica com maior proporção de área úmida o trabalho erosivo é menor.

\section{REFERÊNCIAS}

AGRITEMPO - Sistema de monitoramento agrometeorológico. Dados meteorológicos - Júlio de Castilhos: dados históricos. Disp. em: <http://www.agritempo.gov.br/cnpt/elnino/ mensal.php>. Acesso em: 16 de fevereiro de 2013.

ANDRADE, N.L.R DE; XAVIER, F.V.; ALVES, É.C.R. DE F.; SILVEIRA, A.; OLIVEIRA, C.U. R. DE. Caracterização morfométrica e pluviométrica da Bacia do Rio Manso-MT. Geociências, v. 27, n. 2, 2008.

BIAS, E. DE S.; PIVEL, L.; GUEDES, S.C.; ROCHA, C.R. Análise da eficiência da vegetação no controle do escoamento superficial: Uma aplicação na Bacia Hidrográfica do Rio São Bartolomeu, DF. Geociências, v. 31, n. 3, 2012.

BRESSIANI, D.A. DE; GASSMAN, PHILIP. W.; FERNANDES, J. G.; POSPISSIL GARBOSSA, L.H.; SRINIVASAN, R.; BONUMÁ, N.B.; MENDIONDO, E.M. Review of Soil and Water Assessment Tool (SWAT) applications in Brazil: Challenges and prospects. International Journal of Agricultural and Biological Engineering, v. 8, p. 9-35, 2015.

D'AGOSTINI, L.R. \& MARTINI, L.C.P. Um coeficiente de regularização da vazão: quantificando a qualidade de condições de superfícies. Revista Brasileira de Recursos Hídricos. v .5, n. 2, p. 27-37, 2000.

D'AGOSTINI, L.R. Erosão: o problema mais que o processo. Universidade Federal de Santa Catarina, 1999. 131p.

DENARDIN, J.E.; KOCHHANN, R.A.; FLORES, C.A.; FERREIRA, T.N.; CASSOL, E.A.; MONDARDO, A.; SCHWARZ, R.A. Manejo da enxurrada em sistemas de plantio direto. Porto Alegre: Fórum Estadual de Solo e Água, 2005, 88p.

GARBOSSA, L.H.P.; VASCONCELOS, L.R.C.; LAPA, K.R.; BLAINSKI É.; PINHEIRO, A. The use and results of the Soil and Water Assessment Tool in Brazil: A review from 1999 until 2010. International SWAT. Toledo, 2011, 27 p. In: CONFERENCE \& WORKSHOPS, 2011.

GUBIANI, P.I; VAN LIER, Q. DE J.; DRESCHER, M.S., MEZZOMO, H.C.; VEIGA, C.M.C. Relação entre densidade do solo e conteúdo de água em repetidos ciclos de contração e expansão em um Latossolo. Revista Brasileira de Ciência do Solo, v. 39, p.100-108, 2015.

LANZANOVA, M.E.; NICOLOSO, R. S.; LOVATO, T.; ELTZ, F.L.F.; AMADO, T.J.C.; REINERT, D.J. Atributos físicos do solo em sistema de integração lavoura-pecuária sob plantio direto. Revista Brasileira de Ciência do Solo, v. 31, p. 1131-1140, 2007.

LIMA, G.C.; SILVA, M.L.N.; CURI, N.; SILVA, M.A. da, OLIVEIRA, A.H.; AVANZI, J.C., FREITAS, D.A. F. de. Estimativa do potencial de recarga na sub-Bacia das Posses, Extrema (MG), em função dos atributos fisiógraficos, pedológicos e topográficos. Geociências, v. 32, n. 1, 2013.

MACHADO, A.A. Sistema de análise estatística para Windows (WINSTAT). Pelotas, Universidade Federal de Pelotas, 2001.

MIQUELONI, D.P.; GIANELLO, E.M.; BUENO, C.R.P. Variabilidade espacial de atributos e perda de solo na definição de zonas de manejo. Pesquisa Agropecuária Tropical, v. 45, n. 1, p. 18-28, 2015.

MIOTO, C.L. RIBEIRO, V. DE O.; SOUZA, D.M. DE Q.; PEREIRA, T.V.; ANACHE, J.A.A.; FILHO, A.C.P. Morfometria de Bacias Hidrográficas Através de SIGs Livres e Gratuitos. Anuário do Instituto de Geociências. v. 37, n. 2, 2014.

ODONGO, V.O.; ONYANDO, J.O.; MUTUA, B.M.; VAN OEL, P.R.; BECHT, R. Sensitivity analysis and calibration of the Modified Universal Soil Loss Equation (MUSLE) for the upper Malewa Catchment, Kenya. International Journal of Sediment Research, v. 28, p. 368-383, 2013.

OLIVEIRA, L.F.C. DE. CALIL, P.M.; RODRIGUES, C.; KLIEMANN, H.J.; OLIVEIRA, V.Á. Potencial do uso dos solos da bacia hidrográfica do alto rio Meia Ponte, Goiás. Revista Ambiente \& Água, v. 8, n. 1, 2013.

PEREIRA, V.R. \& FILHO, J.T. Identificação das áreas susceptíveis aos processos erosivos em duas bacias do Sistema Cantareira por meio de diferentes cenários. Acta Scientiarum. Agronomy, v. 31, n. 1, p. 155-163, 2009.

PERAZZOLI, M; PINHEIRO, A; KAUFMANN, V. Efeitos de cenários de uso do solo sobre o regime hídrico e produção de sedimentos na bacia do Ribeirão Concórdia- SC. Revista Árvore. v. 37, n. 5, 2013.

ROCHA, R.M. LUCAS, A.A.T.; ALMEIDA, C.A.P. de; NETO, E.L.M.; NETTO, A. DE O.A. Caracterização morfométrica da sub-bacia do rio Poxim-Açu, Sergipe, Brasil. Revista Ambiental \& Água, v. 9, n. 2, 2014

SANTOS, E.H.M. DOS; GRIEBELER, N.P.; OLIVEIRA, L.F.C. de. Relação entre uso do solo e comportamento 
hidrológico na Bacia Hidrográfica do Ribeirão João Leite. Revista Brasileira de Engenharia Agrícola e Ambiental, v.14, p.826-834, 2010.

SOUZA, R. M.S.; SOUZA, E. S. DE; ANTONIO, C.D.A.; LIMA, J.R. DE S. Balanço hídrico em área de pastagem no semiárido pernambucano. Revista Brasileira de Engenharia Agrícola Ambiental, v. 19, n.5, 2015.

TONELLO, K.C.; DIAS, H.C.T.; SOUZA, A.L. DE; RIBEIRO, C.A.A.S.; LEITE, F.P. Morfometria da bacia hidrográfica da Cachoeira das Pombas, Guanhães - MG. Revista Árvore, v. 30, n. 5, p. 849-857, 2006.

TUCCI, C.E.M. Regionalização de vazão. Porto Alegre: UFRGS, 256 p., 2002,
WISCHMEIER, W.H. \& SMITH, D.D. Predicting rainfall erosion losses - a guide to conservative planning.U.S. Department of Agriculture, Agriculture Handbook, n. 537, 1978, 58p.

ZIECH, A.R.D.; CONCEIÇÃO, P.C.; LUCHESE, A.V.; BALIN, N.M.; CANDIOTTO, G.; GARMUS, T.G. Proteção do solo por plantas de cobertura de ciclo hibernal na região Sul do Brasil. Pesquisa Agropecuária Brasileira. v. 50, n. 5, 2015.

Submetido em 02 de dezembro de 2015 Aceito em 25 de novembro de 2016 